\title{
ERGONOMÍA EN INSTRUMENTACIÓN QUIRÚRGICA
}

\author{
Karen Milena Velasco Rey*
}

\section{Resumen}

Se describe la ergonomía de los profesionales de instrumentación quirúrgica en salas de cirugía, debido a la escasa información existente. La revisión bibliográfica se basó en los datos obtenidos con las palabras clave y operadores booleanos. Se encontraron 51 referencias, 39 de los últimos diez años. La literatura reconoce el riesgo laboral ergonómico de los instrumentadores y señala algunos elementos de la sala operatoria como posibles factores de riesgo. La actividad asistencia en cirugía es cuando más se generan posturas nocivas, asociando algunas especialidades quirúrgicas con el estrés postural y en especial en las laparoscópicas. Se identifican los síntomas músculo esqueléticos más frecuentes en el desempeño laboral así como sus posibles causas, haciendo las recomendaciones preventivas apropiadas para mejorar la ergonomía de estos profesionales en salas de cirugía.

Palabras clave: postura, ergonomía, sala quirúrgica, instrumentador quirúrgico, cirugía, trastornos músculo esqueléticos.

Abreviaturas: IQ, instrumentación quirúrgica, instrumentador (a) quirúrgico (a); RL, riesgos laborales.

\section{ERGONOMICS FOR SURGICAL INSTRUMENT TECNICIANS}

\section{Abstract}

This article describes ergonomics for surgical instrument technicians in operating rooms, for very little information exists about this topic. The bibliographic review was based on data obtained using key words and Boolean operators. Fifty-one references were found, 39 occurring in the last ten years. The ergonomic workplace risks for surgical instrument technicians are acknowledged in literature and include some elements of the operating room as possible risk factors. The assistance in surgery activity is the one which mostly generates unhealthy postures, associated with some surgical specialties postural strain, predominantly during laparoscopic surgeries. The most frequent work-related musculoskeletal symptoms and their possible causes are identified. Similarly, we pose adequate prevention recommendations to improve ergonomics for these professionals in operating rooms.

Key words: posture, ergonomics, operating room, surgical instrument technician, surgery, musculoskeletal disorders.

Fecha recibido: julio II de 2012 - Fecha aceptado: abril 3 de 2013

* Instrumentadora Quirúrgica, Hospital de San José. Instructor Asociado, Fundación Universitaria de Ciencias de la Salud. Bogotá DC; Colombia. 


\section{Introducción}

La ergonomía, según Guillen¹, se define como la ciencia que estudia la relación del ser humano con su entorno, al igual que las posturas apropiadas para realizar las tareas del puesto de trabajo, el manejo de cargas, materiales y movimientos repetitivos, entre otros aspectos. Su objetivo es eliminar o reducir la presencia de fatiga o alteraciones producidas por sobrecarga física durante el desarrollo de las actividades; para ello desarrolla una serie de soluciones destinadas a mejorar las condiciones buscando aumentar la satisfacción y el rendimiento en su lugar de desempeño.

Vieira $^{2}$ asegura que una estación de trabajo ideal en cualquier campo laboral es aquella que permite realizar cambios posturales para llevar a cabo las tareas en forma cómoda y segura, ya que influye de manera directa sobre la carga física a la que está expuesto.

La literatura y los estudios sobre ergonomía en el trabajo del profesional de IQ son escasos, tal vez debido a la ubicación de esta profesión dentro de la enfermería en la mayoría de países del mundo. En Colombia se ejerce la profesión independiente de la enfermería y su campo laboral está principalmente asociado con el área asistencial, en donde a diario se ven sometidos a altos niveles de exigencia y estrés que pueden generar características puntuales en sus posturas, afectando a corto, mediano o largo plazo su salud y sus niveles de satisfacción laboral.

Los profesionales de IQ por lo regular desarrollan sus actividades laborales sin poder reconocer si las posturas que han adoptado en diversos procedimientos quirúrgicos han sido influenciadas por las condiciones técnicas de las cirugías, sus características personales o sus propias condiciones de trabajo, con desconocimiento acerca del riesgo que representan algunas en el desarrollo de desórdenes músculo esqueléticos.

Nogareda ${ }^{3}$ dice que algunas posturas resultan nocivas para la salud debido a que generan una carga estática en el sistema músculo esquelético de la persona, porque la circulación de la sangre y el metabolismo de los músculos disminuye, haciendo que la eficacia sea baja y que se produzca una constricción local muscular y la consecuente fatiga; en casos de larga duración, esta constricción podría llegar a provocar trastornos o patologías relacionados con el trabajo.

Para Kant ${ }^{4}$ es importante determinar las posturas de trabajo del equipo quirúrgico e identificar las actividades laborales que pueden causar estrés en los miembros del mismo, debido a que tiene importantes repercusiones sobre la predisposición, desencadenamiento y agudización de alteraciones y lesiones en el organismo. Por lo tanto, la adopción de determinadas posturas durante largos períodos debe someterse a revisiones ergonómicas cuidadosas, para lograr una mejor adaptación del sujeto a sus tareas y prevenir futuras disfunciones.

Es por lo anterior que esta revisión de la literatura busca identificar el riesgo laboral ergonómico en los profesionales de IQ, así como la revisión de los elementos de una sala de cirugía y su relación con las posturas adoptadas por los IQ durante cirugías a cielo abierto y las mínimamente invasivas. También se busca identificar los desórdenes músculo esqueléticos más relevantes que se pueden desarrollar en estos profesionales a causa de las malas posturas y las posibles alternativas que ofrecen los autores para mejorar su ergonomía en salas de cirugía.

\section{Métodos}

Se realizó una búsqueda bibliográfica en la base de datos de libre acceso PUBMED y en las siguientes bases científicas de acceso restringido: ELSEVIER, MD CONSULT, HINARI, EBSCO HOST, SPRINGER LINK, SCIENCE DIRECT y DYNAMED. Se encontraron 51 artículos relevantes, de los cuales 45 en inglés, cinco en español y uno en polaco. Del total revisado solo 25 se habían publicado en los últimos cinco años, lo que evidencia un escaso abordaje del tema. 


\section{Resultados}

\section{Riesgos laborales en instrumentación quirúrgica}

En el estudio del personal de IQ, desarrollado por Mo$\mathrm{ran}^{5}$, el RL se definió como la probabilidad de que un objeto, material, sustancia o fenómeno cause perturbaciones en la integridad físicao en la salud mental del trabajador; en el ámbito hospitalario las áreas en donde son más evidentes los RL son los servicios de urgencias, laboratorios clínicos y salas de cirugía.

Morán ${ }^{5}$ identifica cuatro RL en el ejercicio profesional de la IQ que son en orden de importancia: biológico, físico, químico y ergonómico. El primero se refiere al contacto con fluidos corporales de pacientes; el físico por el trabajo en condiciones de altas temperaturas y humedad con sistemas deficientes de ventilación y de recambio de aire, exposición a radiaciones ionizantes de rayos $\mathrm{X}$, humo proveniente de los electrocauterios en funcionamiento y el ruido ocasionado por el uso de motores, sierras y sistemas de succión. En cuanto al químico ocurre cuando entran en contacto con sustancias antisépticas como la yodopovidona y el gluconato de clorhexidina, durante el lavado quirúrgico de manos. El riesgo ergonómico es evidente cuando aparecen molestias, contracturas o procesos inflamatorios en su sistema músculo esquelético, debido a los tiempos prolongados de cirugía en los que deben permanecer de pie en posturas fijas, movilizar equipos pesados y ayudar a sostener o trasladar pacientes en algunas ocasiones. Todas las actividades suceden sin tener períodos de descanso y además con movimientos constantes con el riesgo de resbalar o caer.

\section{Elementos del quirófano y sus efectos en la ergonomía}

La mayoría de hospitales y clínicas cuentan con unidades quirúrgicas, las cuales a su vez están conformadas por salas de cirugía dotadas de elementos que permiten realizar intervenciones de diferente nivel de complejidad, cuyo objetivo es garantizar la seguridad en la atención del paciente y proporcionar un ambiente seguro y eficaz para quienes allí trabajan.
Según Matern ${ }^{6}$ en su estudio sobre seguridad, riesgos y ergonomía en salas de cirugía, uno de los elementos más importantes del quirófano es la mesa operatoria, la cual debe ser estable para mantener el soporte de los pacientes y ajustable en su ancho y alto, con el fin de que los miembros del equipo quirúrgico puedan estar cómodos durante los diferentes tipos de cirugías y eviten las elevaciones excesivas de los brazos y las torsiones forzadas del tronco.

En este mismo estudio Matern ${ }^{6}$ describe que por lo regular los quirófanos cuentan con una o dos lámparas cielíticas de igual o diferente tamaño, suspendidas del techo a través de brazos flexibles que permiten su movilidad y manipulación. En ocasiones durante su movilización intraquirúrgica, pueden provocar golpes o lesiones en la cabeza de algunos miembros del equipo quirúrgico estéril o incluso, proporcionarles una iluminación insuficiente del campo operatorio, obligándolos a adoptar posturas forzadas en busca de una mejor visualización del mismo.

Otro elemento importante en el estudio fue la presencia de conexiones de algunos equipos utilizados encirugía, como las unidades para electrocauterios, los sistemas de succión, los enlaces de y hacia las torres de laparoscopia, así como también las conexiones para el funcionamiento de motores o sierras. Al igual que otros autores como Berguer ${ }^{7}$ y Van Veelen ${ }^{8}$, aseguran que estos elementos o más bien sus cables suspendidos, obstaculizan o limitan la movilidad dentro del quirófano y pueden causar tropiezos o caídas en el personal sin mencionar que a menudo se ven obligados a flexionar sus miembros inferiores para elevarse sobre los mismos.

El instrumental quirúrgico utilizado en las cirugías a cielo abierto convencionales, según la descripción hecha por Matern ${ }^{6}$, no suele generar ningún tipo de molestias en quienes lo manipulan, aunque en ocasiones, cuando algunos miembros del equipo quirúrgico deben sostener por tiempos prolongados diversas clases de separadores no autoestáticos, es posible que puedan presentar síntomas de incomodidad, dolor, entumecimiento en las manos, zonas de presión, neuropatías, calambres o que puedan adoptar posturas 
inadecuadas al nivel de los brazos. En cirugías mínimamente invasivas, el instrumental especializado puede producir también zonas de presión, neuropatías y falta de sensibilidad al manipular las estructuras anatómicas, aunque este tipo de molestias se presentan más en los cirujanos.

Por último en el estudio de Matern ${ }^{6}$ se evaluaron también las posturas de trabajo durante el desarrollo de 425 cirugías. El 84\% de estas fueron consideradas por los miembros del equipo quirúrgico como incómodas y dolorosas y la mayoría de sus molestias se evidenciaron en la espalda en $85 \%$, en el cuello $60 \%$ y a nivel de los hombros y los miembros superiores $39 \%$. La cantidad de personas dentro del campo estéril en los procedimientos se consideró como un factor de riesgo para desarrollar estas molestias, debido a que se obstaculizaban entre sí cuando cada uno intentaba cumplir con sus tareas.

\section{Posturas adoptadas por el IQ durante el desarrollo de su trabajo}

Según el estudio de posturas de trabajo estáticas y dinámicas del equipo quirúrgico desarrollado por $\mathrm{Kant}^{4}$, en una jornada laboral de ocho horas los IQ deben realizar el lavado quirúrgico de manos, asistencia en cirugía, preparación del microscopio y labores administrativas, adoptando posturas de predomino estático. Otras actividades que llevan a cabo son el lavado y organización del instrumental, la espera entre cada procedimiento y actos varios en donde las posturas adoptadas son en esencia dinámicas.

En este estudio, Kant ${ }^{4}$ también asegura que los IQ dedican el $78 \%$ del tiempo total de su turno de trabajo a desarrollar la actividad laboral de "asistencia en cirugía" y es ahí en donde adoptan la mayoría de las posturas nocivas, debido a que deben inclinar la cabeza hacia adelante y los lados, así como inclinar y hacer torsión de la espalda hacia adelante. Estas posturas en particular causan una carga que puede tener un efecto perjudicial en el sistema músculo esquelético a corto plazo.
En otro estudio sobre evaluación ergonómica del área de trabajo del IQ hecho por Gerbrands ${ }^{9}$, se puntualizaron las tareas que desarrollan estos profesionales de la salud durante la asistencia en cirugía, como hacer recuento de compresas y material intraquirúrgico, manejar el instrumental y los materiales necesarios para la cirugía, visualizar el campo operatorio, suministrar el instrumental y recogerlo, realizar labores de ayudantía quirúrgica mientras instrumenta, suministrar y en ocasiones utilizar el electrobisturí de la misma manera que el sistema de succión, entregar material de patología a la circulante de la sala y recibir elementos estériles por parte de la misma. Resalta que los IQ llevan a cabo todas estas tareas dentro de su campo estéril que comprende únicamente el espacio que hay entre la cintura y el pecho, lo cual limita en forma significativa sus rangos de movimiento.

Gerbrands ${ }^{9}$ describe también que los IQ como norma trabajan con dos mesas de reserva localizadas al lado o detrás, así como la mesa de Mayo por encima de la superficie de la mesa operatoria, pero que no siempre queda al frente del IQ. Esta distribución causa torsión de la parte superior del cuerpo, el giro del cuerpo entero o realizar inclinaciones hacia los lados para alcanzar los instrumentos.

En el análisis ergonómico sobre enfermería instrumentista hecho por Vilela ${ }^{10}$, se menciona que la altura de la mesa operatoria casi siempre la define el cirujano y a menudo difiere de la óptima para el IQ. Estas distorsiones en la altura de la mesa operatoria pueden generar dos diferentes tipos de problemas para los IQ: si es excesiva los obliga a elevar los brazos por encima del nivel de los hombros y por el contrario, si está muy baja hacen numerosas inclinaciones del tronco hacia adelante.

Otro estudio hecho por Kutagowska ${ }^{11}$ en el 2009 acerca de la carga del sistema músculo esquelético en las enfermeras de quirófano y sus determinantes, sugiere que las posturas usuales del IQ durante sus actividades laborales son normales y naturales en un $89 \%$ y por tanto no necesitan ser corregidas. 
Por otra parte, Kant ${ }^{4}$ en su estudio también sugiere que las especialidades quirúrgicas pueden influenciar en la adopción de determinadas posturas. En procedimientos de cirugía general, por ejemplo, es posible observar niveles superiores de estrés ergonómico en las regiones de los hombros, la espalda y el cuello, en comparación con que surgen durante intervenciones de oído, nariz y cuello. Los primeros se caracterizan por tener la espalda inclinada o con torsión hacia adelante y por permanecer de pie apoyándose sobre una pierna más que otra, a diferencia de los segundos que se caracterizan por permanecer sentados la mayor parte del tiempo. En relación con las posturas de los brazos, $\mathrm{Kant}^{4}$ no reporta diferencias significativas entre las cirugías de oído, nariz y cuello y los procedimientos de cirugía general, aunque si menciona la posibilidad de que se desarrolle menos estrés postural en las primeras debido a que se llevan a cabo en el 57.6\% del tiempo del turno total de trabajo, mientras que los procedimientos de cirugía general consumen el $68.1 \%$.

Mirbod $^{12}$ por otro lado, en su estudio sobre quejas subjetivas de ortopedistas y cirujanos generales, plantea que durante procedimientos ortopédicos se sufre mayor incomodidad con síntomas a nivel de los hombros, rigidez en la espalda baja, dolor en el cuello y entumecimiento de los dedos, que pueden estar asociados con el uso de chalecos de plomo que protegen contra la radiación durante los procedimientos, pero producen una carga biomecánica considerable sobre el cuerpo.

Engels ${ }^{13}$ menciona que tanto en quirófanos de ortopedia como de urología, a pesar de que la carga postural del trabajo y la duración de los procedimientos en cada especialidad son diferentes, los IQ desarrollan algunas de sus tareas adoptando posturas "pobres" y que el $20 \%$ causan una carga que puede tener efecto perjudicial en el sistema músculo esquelético a corto plazo, lo que resulta nocivo para estos profesionales debido a que trabajan en estas condiciones una gran parte de su turno de trabajo diario.

En un estudio sobre procesos pelvianos periarticulares en IQ de quirófano, realizado por Idoate ${ }^{14}$, se observó que en especialidades como cirugía cardiovascular y de tórax, los IQ por lo regular se ubican al lado derecho del cirujano haciendo uso de un elevador o banquillo para estar acordes con la altura de la mesa operatoria y estas condiciones suelen obligarlos a realizar movimientos de flexión, rotación y flexión lateral del tronco, así como a descargar todo el peso de su cuerpo sobre una de sus dos piernas mientras mantienen sus brazos por encima del nivel de los hombros al entregar o recibir instrumental. Según Idoate ${ }^{14}$, esto pueden resultar muy incómodo y provocar una sobrecarga postural a corto plazo, en especial si no existen períodos de pausa o descanso durante el procedimiento.

En relación con las cirugías intrabdominales e intratorácicas, Gerbrands ${ }^{9}$ aconseja que para obtener una visualización del campo operatorio sin obstrucciones en una postura de pie neutral, el ángulo de visión más recomendable debe estar entre 10 y 25 grados por debajo del plano horizontal y hasta 30 grados para girar hacia la derecha o izquierda en el plano sagital.

\section{Ergonomía del IQ en cirugías mínimamente invasivas}

De acuerdo con Berguer ${ }^{7}$, durante el desarrollo de cirugías mínimamente invasivas los miembros del equipo quirúrgico experimentan altos niveles de frustración y tensión. También Vereczkei ${ }^{15}$ argumenta que la intervención laparoscópica no es ergonómica y por lo tanto incrementa el estrés físico y mental de quienes la llevan a cabo.

En el estudio hecho por Van Veelen ${ }^{16}$ uno de los principales problemas ergonómicos para los IQ en la asistencia de cirugías laparoscópicas es la adopción de posturas nocivas asociadas con tres factores: la posición del monitor, altura de la mesa operatoria y posturas estáticas prolongadas asumidas durante los procedimientos.

Berguer $^{7}$ asegura que el uso de monitores en cirugía laparoscópica disminuye la carga física en los músculos de los hombros de los miembros del equipo quirúrgico y les permite ver las cavidades de los pacientes manteniendo una posición recta de la espalda, a pesar de que disminuye la movilidad del resto del cuerpo; Sari $^{17}$ y Van Det ${ }^{18}$ afirman que la posición del monitor 
puede generar posturas estáticas que afectan las áreas del cuello, la espalda y los hombros. La posición correcta del monitor es cuando permite una visión recta hacia adelante con una ligera angulación hacia abajo. Siguiendo la recomendación de Van Det ${ }^{18}$, Seghers ${ }^{19}$ sugiere que esta debe estar entre tres y 16 grados por debajo del plano horizontal. Van Det $^{20}$ en otro estudiotambién aconseja evitar posiciones elevadas del monitor por encima del nivel de los ojos y en aquellos de 19 pulgadas, propone una distancia entre 80 y 120 $\mathrm{cm}$ a partir del campo operatorio, aunque aclara que puede variar de acuerdo con el tamaño del monitor y la resolución de las imágenes. En desacuerdo con lo anterior, Szeto ${ }^{21}$ asevera que la altura de un monitor de cirugía laparoscópica se debe encontrar al nivel de los ojos de los miembros del equipo quirúrgico o incluso un poco más arriba, a pesar de que Matern ${ }^{22}$ señala que cuando los monitores se encuentran en la parte superior de estantes o torres de laparoscopia, su ajustabilidad es nula y desfavorecen a las personas de menor estatura del equipo quirúrgico, porque deben mantener sus cabezas extendidas hacia arriba para ver. Vereczkei ${ }^{15}$ y Papp $^{23}$ argumentan que cuando los monitores están al centro a nivel de la cabeza del paciente, los operadores tienden a bloquear la visibilidad entre si y los IQ se ven obligados a inclinarse hacia adelante y a rotar la cabeza y el torso para poder verlo por lo que recomiendan colocarse al lado derecho del paciente o usar otro monitor al frente de los IQ para que puedan mantener posturas neutras.

En cuanto a la altura de la mesa operatoria en cirugía laparoscópica, Nguyen $^{24}$ afirma que es un factor que afecta el estrés músculo esquelético; Van Veelen ${ }^{16}$ asegura que dicha altura influye en forma directa en el desempeño de los brazos de las personas que conforman el equipo quirúrgico y recomienda ajustarla con la de los codos de todo el equipo. Estos según Szeto ${ }^{21}$, se deben mantener angulados a 90 o 100 grados durante los procedimientos. Berquer ${ }^{25}$ propone que la altura de la mesa debe ser más baja que la utilizada en cirugías a cielo abierto y que se debe revisar que los mangos de los instrumentos laparoscópicos estén al nivel de los codos de los miembros del equipo quirúrgico o $10 \mathrm{~cm}$ por debajo. Por el contrario, Manasnayakorn ${ }^{26}$ argumenta que la altura adecuada es la que permite que los mangos de los instrumentos laparoscópicos estén al nivel de los codos de los miembros del equipo quirúrgico o $5 \mathrm{~cm}$ por encima del nivel de estos, ya que según su estudio la altura promedio de los codos se encuentra entre 92.86 y $125 \mathrm{~cm}$; Berguer ${ }^{25}$ en otra parte de su artículo menciona que la superficie de la mesa operatoria debe estar entre 64 y $77 \mathrm{cms}$ a partir del suelo, pero Manasnayakorn ${ }^{26}$ de nuevo lo contradice diciendo que es entre 57 y $99 \mathrm{~cm}$ a partir del suelo, debido a la altura de la pared abdominal del paciente, la cual por la insuflación aumenta de 31 a $36 \mathrm{~cm}$ la superficie de trabajo para todos los miembros del equipo quirúrgico; Wauben ${ }^{27}$ en su estudio de aplicación de lineamientos ergonómicos durante cirugía mínimamente invasiva, señala que de 2.192 cuestionarios respondidos, 64\% reportaron malestares en el cuello y $77 \%$ en los hombros, debido a que la superficie real de trabajo fue mayor a la altura de la mesa operatoria, casi siempre por el tamaño propio del paciente y la insuflación de la cavidad, lo cual los obligó a desarrollar todo el procedimiento con los hombros y brazos en posiciones elevadas. Para concluir con las discrepancias en este tema, Van Veelen ${ }^{8}$ propone ajustar la mesa operatoria según la persona más alta del equipo quirúrgico, justificándose en que las personas de menor estatura pueden usar elevadores o banquillos.

En lo concerniente a las posturas estáticas en cirugía laparoscópica, varios autores como Berguer ${ }^{7,28,29}$, Van Det $^{18}$, Vieira ${ }^{2}$, Nguyen ${ }^{24}$ yMatern $^{22}$ coinciden en que los IQ experimentan síntomas como estrés a nivel músculo esquelético, fatiga rápida, calambres, dolor muscular y malestar durante la asistencia de cirugías mínimamente invasivas, debido a la adopción de posturas estáticas e incómodas por tiempos prolongados, en especial en la espalda y el cuello, así como también debido a la realización de movimientos repetitivos a nivel de los brazos. Como norma, en estos largos períodos la espalda se mantiene en posición recta, mientras que la cabeza y el cuello suelen permanecer centradas o inclinadas, sin mencionar la restricción de movimientos que hay en el resto de zonas del cuerpo. Van Veelen ${ }^{8-16}$ complementa lo anterior al afirmar que el malestar que experimentan los IQ a nivel del cuello, los hombros y la espalda, se debe también al hecho de permanecer de pie durante tiempo prolongado, lo que genera una 
obstrucción del flujo sanguíneo en las piernas y aumento de la presión en la columna vertebral.

Por el contrario, de acuerdo con los estudios desarrollados por Papp ${ }^{23}$ y Vereczkei ${ }^{15}$, durante procedimientos de colelap, en donde la duración promedio oscila entre 2.768 y 2.931 segundos, los movimientos de los profesionales de IQ pueden ser tanto de naturaleza estática como dinámica. Los primeros se llevan a cabo en el $22 \%$ del tiempo total del procedimiento y se reconocen en los pasos de la mitad de la cirugía, es decir, durante la preparación del triángulo de Callot, el pinzamiento de la arteria y el conducto cístico y la disección de la vesícula biliar; durante estos pasos los IQ suelen concentrarse en el curso de la cirugía mirando hacia el monitor y las posturas constantes del tronco junto con los movimientos de los brazos, son interrumpidos por cortos intervalos de cambio de los instrumentos laparoscópicos. En cambio los movimientos dinámicos, se llevan a cabo en el $78 \%$ del tiempo del procedimiento y se reconocen en los pasos del comienzo y el final de la cirugía, es decir, durante la insuflación, colocación de trocares, extracción de la vesícula biliar, coagulación, lavado más succión del lecho hepático y el cierre de las heridas.

Para concluir, otros autores como Hemal ${ }^{30}$, Supe ${ }^{31}$, Reddy $^{32}$ y Klein ${ }^{33}$, señalan que el uso de salas de cirugía laparoscópicas adecuadas y modernas junto con superiores programas especiales de entrenamiento, resultan esenciales para mejorar la ergonomía de todos los miembros del equipo quirúrgico en relación con la eficiencia, el balance postural, la seguridad y el bienestar del equipo quirúrgico, al igual que para reducir en forma drástica la fatiga quirúrgica, las quejas a nivel músculo esquelético y la incidencia de lesiones por estrés repetitivo.

\section{Desórdenes músculo esqueléticos asociados con problemas ergonómicos en IQ}

Diversos autores han coincidido en que durante el ejercicio profesional de la IQ se desarrollan desórdenes músculo esqueléticos puntuales asociados con malas prácticas ergonómicas en los sitios de trabajo. Por ejemplo, Liang ${ }^{34}$, Meijsen $^{35}$, Szeto $^{36}$, Bos $^{37}$ y Chan ${ }^{38}$, entre muchos otros, afirman que los desórdenes músculo esqueléticos más comunes son el dolor de espalda ${ }^{39-43}$ seguido por el del cuello, hombros y miembros inferiores, al igual que la hinchazón de los tobillos y los pies, ${ }^{44-45}$ la degeneración de los discos intervertebrales y su consecuente formación de hernias discales, ${ }^{46}$ las osteopatías dinámicas del pubis, las peritrocanteritis ${ }^{14}$ y la aparición de várices.

La literatura señala que las principales causas de estos desórdenes son los movimientos repetitivos sin hacer cambios periódicos de posición, adoptar posturas estáticas no neutrales o inclinadas y con torsión, trabajar de pie por tiempo prolongado, inclinar la columna más de 45 grados para recoger algún elemento del suelo, realizar actividades que implican jalar o empujar, trabajar en condiciones climáticas desfavorables, manipular cargas pesadas o usar chalecos de plomo por varias horas, además de manejar altos niveles de estrés, ayudar a la transferencia de los pacientes, sostener instrumentos o retractores en algunas cirugías, laborar un mayor número de horas o asistir a un alto número de procedimientos, tener bajos niveles de satisfacción laboral e incluso la relación deficiente con compañeros o colegas al carecer del apoyo de estos en algunas actividades.

Autores como Walker-Bone ${ }^{47}$, Stock ${ }^{48}$, Latko ${ }^{49}$ y Van $\mathrm{Rijn}^{50}$, también sugieren que otros desórdenes músculo esqueléticos que se presentan a menudo en el desempeño de los IQ son la incomodidad a nivel de las muñecas y las manos, los desórdenes de miembros superiores, tendinitis en la parte distal de los brazos, síndrome del túnel carpiano, epicondilitis de la región medial y lateral y los síndromes del túnel cubital y radial. Según los autores, se deben en esencia al manejo constante de cargas pesadas de más de $20 \mathrm{k}$ o de herramientas de más de $1 \mathrm{k}$, así como a la realización de movimientos repetitivos por más de dos horas al día, desarrollo de trabajos forzados, pobre control sobre las actividades propias del trabajo, laborar bajo condiciones de vibración constante, las flexiones repetitivas a nivel de los codos y mantener las manos en posiciones estáticas, inclinadas o con torsión mientras ejecutan movimientos de precisión durante gran parte del día de trabajo. 
Hull $^{51}$, Meijsen ${ }^{35}$ Toffola $^{40}$, Yip ${ }^{41}$ y Liang ${ }^{34}$, plantean en sus estudios una serie de recomendaciones útiles para disminuir los factores de riesgo, como adoptar buenas posturas durante la cirugía limitando así los tiempos de exposición a las posturas nocivas, bien sea sentándose más a menudo, haciendo pausas o apoyando en forma intermitente el peso del cuerpo sobre una y otra pierna, cambiar algunos de los elementos de la sala de cirugía para hacerlos más ergonómicos, mejorar la calidad de las condiciones climáticas dentro del quirófano, utilizar bandejas de instrumental más livianas, acordar con el cirujano la altura óptima de la mesa operatoria, planificar y alternar mejor los deberes del trabajo, organizar las áreas en forma conveniente, crear mejores políticas de prevención del estrés físico, recibir más información, entrenamientos y valoraciones continuas de las posturas de trabajo, y consultar con especialistas en terapia ocupacional.

\section{Conclusiones}

De acuerdo con la literatura revisada en el ejercicio profesional de la IQ en salas de cirugía, se presenta riesgo ergonómico al adoptar posturas nocivas durante tiempos prolongados para cumplir con algunas actividades propias del trabajo.

La literatura afirma que de todos los elementos presentes en una sala de cirugía son la mesa operatoria, las lámparas cielíticas y los cables de algunos equipos, los que más se relacionan con la adopción de posturas incómodas y dolorosas en especial en la espalda, el cuello, los hombros y los miembros superiores.

Algunos autores que desarrollaron estudios específicos en IQ señalan que de las muchas actividades laborales que se efectúan durante un turno, es la "asistencia en cirugía" donde más se adoptan posturas nocivas asociadas con la inclinación hacia adelante y hacia los lados de la cabeza y la espalda. Indican que la mayoría están relacionadas con la altura, forma, disposición y uso de las mesas rutinarias del instrumental y las de reserva, así como la operatoria. Incluso hay quienes aseguran que algunas especialidades quirúrgicas pueden influenciar la adopción de posturas nocivas como cirugía general, toráxica, cardiovascular, ortopedia y urología.

En relación con la cirugía mínimamente invasiva, casi todos los estudios coinciden en que los problemas ergonómicos suelen estar vinculados al posicionamiento del monitor, la altura de la mesa operatoria y las posturas estáticas por los tiempos prolongados de cirugía. La mayoría asegura que el monitor ojalá siempre esté al lado derecho del paciente y que la altura óptima es aquella que permite una visión en línea recta hacia él junto con una pequeña angulación por debajo del plano horizontal de 3 a 16 grados. En cuanto a la mesa operatoria las opiniones difieren debido a que según algunos se debe ajustar a la altura de los codos de los miembros del equipo quirúrgico, otros recomiendan la altura de las manijas de los instrumentos laparoscópicos y otros más consideran que se debe ajustar de acuerdo con el aumento de la altura dada por la pared abdominal del paciente a causa de la insuflación. En lo concerniente a la postura estática por tiempos prolongados, algunos afirman que en las laparoscópicas se adopta mayor cantidad del tipo estático mientras otros aseguran hay mayor adopción de las dinámicas.

Por último, varios autores concuerdan en que la mayoría de los desórdenes músculo esqueléticos asociados con los problemas ergonómicos en los profesionales de IQ son dolor de cuello, hombros y miembros inferiores, hinchazón de los tobillos y de los pies, desarrollo de hernias discales, osteopatías dinámicas del pubis, peritrocanteritis, aparición de várices, incomodidad de las muñecas y las manos, así como desórdenes de miembros superiores como tendinitis en la parte distal de los brazos, síndrome del túnel carpiano, epicondilitis de la región medial y lateral y los síndromes del túnel cubital y radial. Afirman que si los IQ modifican su propio comportamiento adoptando buenas posturas en el trabajo, sobre todo durante cirugía, limitando los tiempos de exposición a posturas nocivas, conseguirán mejorar su ergonomía en forma significativa. 


\section{Referencias}

1. Guillen M. Ergonomía y la relación con los factores de riesgo en salud ocupacional. Rev cubana enfermer. 2006 Dic; 22(4).

2. Vieira ER, Kumar S. Working postures: a literature review.J Occup Rehabil 2004 Jun; 14(2):143-59.

3. Nogareda S, Dalmau I. NTP 452: evaluación de las condiciones de trabajo: carga postural.Madrid, España: INSHT;1997.

4. Kant IJ, de Jong LC, van Rijssen-Moll M, Borm PJ. A survey of static and dynamic work postures of operating room staff. Int Arch Occup Environ Health. 1992; 63(6):423-8.

5. Morán CJ. Occupational hazards of professional nursing in Hospital Sergio E Bernales - Collique operating rooms, February-August 2009. Rev Per Obst Enf. 2009; 5(2):104-9.

6. Matern U, Koneczny S. Safety, hazards and ergonomics in the operating room SurgEndosc.2007 Nov; 21(11):1965-9.

7. Berguer R. Surgery and ergonomics. Arch Surg. 1999 Sep; 134(9):1011-6.

8. Van Veelen MA, Nederlof EA, Goossens RH, Schot CJ, Jakimowicz JJ. Ergonomic problems encountered by the medical team related to products used for minimally invasive surgery. Surg Endosc. 2003 Jul; 17(7):1077-81.

9. Gerbrands A, Albayrak A, Kazemier G. Ergonomic evaluation of the work area of the scrub nurse. Minim Invasive Ther Allied Technol. 2004 Jun; 13(3):142-6.

10. VilelaJA, Diaz T, Sanfeliz A.Analisis ergonomico en enfermeria instrumentista: un enfoque descriptivo. Seguridad y salud en el trabajo. 2003; 24:5-10.

11. Kunagowska E. Musculoskeletal system load in operating room nurses and its determinants. Med Pr. 2009; 60(3):187-95.

12. Mirbod SM, Yoshida H, Miyamoto K, Miyashita K, Inaba R, Iwata H. Subjective complaints in orthopedists and general surgeons. Int Arch Occup Environ Health. 1995; 67(3):179-86.

13. Engels JA, Landeweerd JA, Kant Y. An OWAS-based analysis of nurses' working postures. Ergonomics. 1994 May; 37(5):909-19.

14. Idoate VM, Bravo B, Guergue MC, Berraondo J, Sagues N, Mendaza I. Procesos pelvianos periarticulares en instrumentistas de quirófano. Arch Prev Riesgos Labor. 2002; 5(2):48-52.

15. Vereczkei A, Feussner H, Negele T, Fritzsche F, Seitz T, Bubb H, Horváth OP. Ergonomic assessment of the static stress confronted by surgeons during laparoscopic cholecystectomy. Surg Endosc. 2004 Jul; 18(7):1118-22.

16. Van Veelen MA, Jakimowicz JJ, Kazemier G. Improved physical ergonomics of laparoscopic surgery. Minim Invasive Ther Allied Technol. 2004 Jun; 13(3):161-6.

17. Sari V, Nieboer TE, Vierhout ME, Stegeman DF, Kluivers KB. The operation room as a hostile environment for surgeons: physical complaints during and after laparoscopy. Minim Invasive Ther Allied Technol. 2010 Apr; 19(2):105-9.

18. Van Det MJ, Meijerink WJ, Hoff C, van Veelen MA, Pierie JP. Ergonomic assessment of neck posture in the minimally invasive surgery suite during laparoscopic cholecystectomy. Surg Endosc. 2008 Nov; 22(11):2421-7.

19. Seghers J, Jochem A, Spaepen A. Posture, muscle activity and muscle fatigue in prolonged VDT work at different screen height settings. Ergonomics. 2003 Jun 10; 46(7):714-30.

20. Van Det MJ, Meijerink WJ, Hoff C, Totté ER, Pierie JP. Optimal ergonomics for laparoscopic surgery in minimally invasive surgery suites: a review and guidelines. Surg Endosc. 2009 Jun; 23(6):1279-85

21. Szeto GP, Ho P, Ting AC, Poon JT, Tsang RC, Cheng SW. A study of surgeons postural muscle activity during open, laparoscopic, and endovascular surgery. Surg Endosc. 2010 Jul; 24(7):1712-21

22. Matern U. Ergonomic deficiencies in the operating room: examples from minimally invasive surgery. Work. 2009; 33(2):165-8.

23. Papp A, Feussner H, Seitz T, Bubb H, Schneider A, Horvath OP, Vereczkei A Ergonomic evaluation of the scrub nurse's posture at different monitor positions during laparoscopic cholecystectomy. Surg Laparosc Endosc Percutan Tech. 2009 Apr; 19(2):165-9.

24. Nguyen NT, Ho HS, Smith WD, Philipps C, Lewis C, De Vera RM, Berguer R. An ergonomic evaluation of surgeons' axial skeletal and upper extremity movements during laparoscopic and open surgery. Am J Surg. 2001 Dec; 182(6):720-4.

25. Berquer R, Smith WD, Davis S. An ergonomic study of the optimum operating table height for laparoscopic surgery. Surg Endosc. 2002 Mar; 16(3):416-21.

26. Manasnayakorn S, Cuschieri A, Hanna GB. Ergonomic assessment of optimum operating table height for hand-assisted laparoscopic surgery. SurgEndosc. 2009 Apr; 23(4):783-9. Epub 2008 Jul 16
27. Wauben LS, van Veelen MA, Gossot D, Goossens RH. Application of ergonomic guidelines during minimally invasive surgery: a questionnaire survey of 284 surgeons.SurgEndosc. 2006 Aug; 20(8):1268-74.

28. Berguer R, Rab GT, Abu-Ghaida H, Alarcon A, Chung J. A comparison of surgeons' posture during laparoscopic and open surgical procedures. Surg Endosc. 1997 Feb; 11(2):139-42.

29. Berguer R, Forkey DL, Smith WD. Ergonomic problems associated with laparoscopic surgery. Surg Endosc. 1999 May; 13(5):466-8.

30. Hemal AK, Srinivas M, Charles AR. Ergonomic problems associated with laparoscopy. J Endourol. 2001 Jun; 15(5):499-503.

31. Supe AN, Kulkarni GV, Supe PA. Ergonomics in laparoscopic surgery. J Minim Access Surg. 2010 Apr; 6(2):31-6.

32. Reddy PP, Reddy TP, Roig-Francoli J, Cone L, Sivan B, DeFoor WR, Gaitonde $\mathrm{K}$, Noh PH. The impact of the alexander technique on improving posture and surgical ergonomics during minimally invasive surgery: pilot study. J Urol. 2011 Oct; 186(4 Suppl):1658-62

33. Klein M, Andersen LP, Alamili M, Gögenur I, Rosenberg J. Psychological and physical stress in surgeons operating in a standard or modern operating room. Surg Laparosc Endosc Percutan Tech. 2010 Aug; 20(4):237-42.

34. Liang CA, Levine VJ, Dusza SW, Hale EK, Nehal KS. Musculoskeletal disorders and ergonomics in dermatologic surgery: a survey of Mohs surgeons in 2010. Dermatol Surg. 2012 Feb; 38(2):240-8.

35. Meijsen P, Knibbe HJ. Work-related musculoskeletal disorders of perioperative personnel in the Netherlands. AORN J. 2007 Aug; 86(2):193-208.

36. Szeto GP, Ho P, Ting AC, Poon JT, Cheng SW, Tsang RC. Work-related musculoskeletal symptoms in surgeons.J Occup Rehabil. 2009 Jun; 19(2):175-84.

37. Bos E, Krol B, van der Star L, Groothoff J. Risk factors and musculoskeletal complaints in non-specialized nurses, IC nurses, operation room nurses, and Xray technologists.Int Arch Occup Environ Health. 2007 Jan; 80(3):198-206.

38. Chan OY, Ho SF. Study on musculoskeletal complaints involving the back, neck and upper limbs. Singapore Med J.1998 Aug; 39(8):363-7.

39. Das B, Sengupta AK. Evaluation of low back pain risks in a beef skinning operation. Int J Occup Saf Ergon. 2000; 6(3):347-61.

40. Toffola ED, Rodigari A, Di Natali G, Ferrari S, Mazzacane B. Posture and fatigue among surgeons in the operating room. G Ital Med Lav Ergon. 2009 Oct-Dec; 31(4):414-8

41. Yip VY. New low back pain in nurses: work activities, work stress and sedentary lifestyle. J Adv Nurs. 2004 May; 46(4):430-40.

42. Freitag S, Ellegast R, Dulon M, Nienhaus A. Quantitative measurement of stressful trunk postures in nursing professions. Ann Occup Hyg. 2007 Jun; 51(4):385-95

43. Hansson T, Jensen I. Swedish Council on Technology Assessment in HealthCare (SBU). Chapter6. Sickness absence due to back and neck disorders. Scand J Public Health Suppl. 2004; 63:109-51.

44. Sheikhzadeh A, Gore C, Zuckerman JD, Nordin M. Perioperating nurses and technicians' perceptions of ergonomic risk factors in the surgical environment. Appl Ergon. 2009 Sep; 40(5):833-9.

45. Engels JA, van der Gulden JW, Senden TF, van't Hof B.Work related risk factors for musculoskeletal complaints in the nursing profession: results of a questionnaire survey. Occup Environ Med. 1996 Sep; 53(9):636-41.

46. Karahan A, Kav S, Abbasoglu A, Dogan N. Low back pain: prevalence and associated risk factors among hospital staff. J Adv Nurs. 2009 Mar; 65(3):516-24.

47. Walker-Bone K, Cooper C. Hard work never hurt anyone--or did it? A review of occupational associations with soft tissue musculoskeletal disorders of the neck and upper limb. Ann Rheum Dis. 2005 Aug; 64(8):1112-7.

48. Stock SR. Work place ergonomic factors and the development of musculoskeletal disorders of the neck and upper limbs: a meta-analysis. Am J Ind Med. 1991; 19(1):87-107.

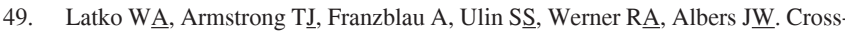
sectional study of the relationship between repetitive work and the prevalence of upper limb musculoskeletal disorders. Am JInd med. 1999 Aug; 36(2):248-59.

50. Van Rijn RM, Huisstede BM, Koes BW, Burdorf A. Associations between workrelated factors and specific disorders at the elbow: a systematic literature review. Rheumatology (Oxford). 2009 May; 48(5):528-36.

51. Hull L, Arora S, Kassab E, Kneebone R, Sevdalis N. Assessment of stress and teamwork in the operating room: an exploratory study. Am J Surg. 2011 Jan; 201(1):24-30. 